\title{
XLIV. On Laplace's Equation
}

John H. Jellett D.D.

To cite this article: John H. Jellett D.D. (1884) XLIV. On Laplace's Equation , Philosophical Magazine Series 5, 18:114, 400-404, DOI: 10.1080/14786448408627613

To link to this article: http://dx.doi.org/10.1080/14786448408627613

曲 Published online: 29 Apr 2009.

Submit your article to this journal 준

Џll Article views: 3

Q View related articles $₫$ 


\section{On Laplace's Equation. By JOHN H. JELLETT,} D.D., Provost of Trinity College, Dublin*.

THE equation upon which Laplace founded his discussion 1 of the problem of attraction has excited, as is well known, a large amount of controversy. No doubt, indeed, appears to exist as to the truth of this equation in the case to which alone Laplace has applied it, nor therefore as to the validity of the method which he has built upon this foundation. But Laplace professes to demonstrate the truth of his equation for a case more general than that which he subsequently considers; and it is in this more general form that its truth has been questioned. My object in the present paper is to endeavour to determine exactly the limits within which Laplace's equation is true, as it appears to me that these limits are somewhat wider than has been generally supposed $\dagger$.

I proceed, in the first place, to give a proof of this equation agreeing substantially with that given by Laplace, and then to consider what conditions or limitations, if any, are necessary to the validity of this proof. Laplace's equation is as follows:-

Suppose the force of attraction to vary as the $n$th power of the distance. Let $\mathrm{V}$ be the potential of the attraction of a nearly spherical body on a point upon its surface. Let $V_{1}$ be the potential (at the same point) of a sphere, touching the surface at the point in question, and separated from it throughout by a small distance, which we may denote by au, $\alpha$ being a small constant quantity, and $u$ a function of the polar angles $\theta, \phi$. Let $\mathrm{A}, \mathrm{A}_{1}$ be the attractions, resolved along the common normal, of the original solid and the sphere respectively. Let also $b$ be the radius of the sphere. Laplace's equation is (Méc. Cél. Livr. 3, chap. ii. sect. 10),

$$
\mathrm{V}=\mathrm{V}_{1}+\frac{2 b}{n+1}\left(\mathrm{~A}-\mathrm{A}_{1}\right) . \quad . \quad . \quad \text {. }
$$

This equation may be put under a form somewhat simpler, and which will enable us to mark more distinctly the limits within which it is true. Let $v$ be the potential, at the point of contact, of the shell included between the original surface and the sphere. Let also $a$ be its normal attraction at the same point. Then

$$
v=\mathrm{V}-\mathrm{V}_{1}, \quad a=\mathrm{A}-\mathrm{A}_{1},
$$

* Communicated by the Author.

$\uparrow$ "It may be taken, I think, as universally admitted that the equation cannot be considered established if $n$ is negative and numerically greater than 2" (Todhunter, "History of the Mathematical Theories of Attraction and the Figure of the Earth,' vol. ii. p. 263). 
and the equation becomes

$$
(n+1) v=2 b a ; \quad . \quad . \quad . \quad .
$$

or, as it may be otherwise written,

$$
(n+1) v=2 b \frac{d v}{d r}
$$

where $r$ is the distance from the centre of the sphere of a point situated on the radius through the point of contact.

To prove this, Laplace assumes in the first place that the thin shell lying between the sphere and the original surface may be replaced by a "distribution" of matter (to use Gauss's expression) on the spherical surface itself. This assumption is only approximately true, inasmuch as it neglects the differences of distance among the particles sitnated on the same radius vector. The degree of the approximation will be considered further on.

Let $\mathrm{P}$ be the point of contact, and $\mathrm{Q}$ any other point on the surface of the sphere. Taking the origin, $O$, at the centre of the sphere, put $\mathrm{POQ}=\theta, \mathrm{PQ}=p$, and let $\epsilon$ be the density of the distribution at the point $Q$. Then evidently

But

$$
\begin{aligned}
(n+1) v & =b^{2} \int_{0}^{\pi} \int_{0}^{2 \pi} \epsilon \rho^{n+1} \sin \theta d \theta d \phi, \ldots . \\
a & =b^{2} \int_{0}^{\pi} \int_{0}^{2 \pi} \epsilon \rho^{n} \cos \mathrm{QPO} \sin \theta d \theta d \phi .
\end{aligned}
$$

whence

$$
20 \mathrm{P} \cos \mathrm{QPO}=2 \mathrm{PQ}=\rho,
$$

$$
2 b a=b^{2} \int_{0}^{\pi} \int_{0}^{2 \pi} \epsilon \rho^{n+1} \sin \theta d \theta d \phi . . .
$$

Hence $(n+1) v=2 b a$; and Laplace's equation is proved, if the foregoing integration be legitimate. It is of course only approximately true, the degree of approximation being the same as that of the assumption made in the beginning, namely that a distribution on the spherical surface may be substituted for the thin shell.

We have then to inquire, in the first place, what conditions are necessary to the validity of the integration indicated in (4) and (5), and, secondly, to what degree of approximation is the result true.

In reply to the first question, it may be said that it is sufficient to ensure the validity of the process of integration that the function to be integrated remain finite within the limits of integration. Thus, if the integral be $\iint U d \theta d \phi$, it is 
sufficient that $U$ remain finite for all values of $\theta$ and $\phi$ included within the limits of integration. Applying this principle to the integral in (4) and (5), we see that the process of integration will be legitimate if $\epsilon \rho^{n+1} \sin \theta$ remain finite within the limits of integration. Now it is evident that this expression can become very great only when $n+1$ is negative and $\rho(=\mathrm{PQ})$ is very small. But if $\rho$ is small, it is plain that $\theta$ is small. It will be sufficient, therefore, to consider the limiting value of $\epsilon \rho^{n+1} \sin \theta$ for very small values of $\theta$. Now $\epsilon$, the density of distribution, is proportional to the thickness of the shell at each point. Hence if a line be drawn from $O$, the centre of the sphere, cutting the sphere and the original surface in $Q, Q^{\prime}$ respectively, the density of distribution, $\epsilon$, at the point $Q$, will be proportional to $\mathrm{QQ}^{\prime}$. Now, observing that $\mathrm{OP}$ is a normal to both surfaces, we see that, if $\operatorname{POQ}(=\theta)$ be a small angle, $\mathrm{QQ}^{\prime}$ cannot be of an order lower than $\theta^{2}$. For both $\mathrm{OQ}$ and $O Q^{\prime}$ differ from $O P$ by a quantity of this order. Hence the greatest value which $\epsilon$ can have at the point $Q$ is $K \theta^{2}$, where $\mathrm{K}$ is finite. Again, $\rho=2 b \sin \frac{1}{2} \theta=b \theta$ nearly, and $\sin \theta=\theta$, to the same order. Hence $\epsilon \rho^{n+1} \sin \theta=K \theta^{n+4}+$ higher terms. It is evident, then, that $\epsilon \rho^{n+1} \sin \theta$ will not be infinite for $\theta=0$ unless $n+4<0$. Laplace's equation is therefore true for all positive values of $n$, and for all negative values (except $n=-1$ ) which are not numerically greater than 4. The equation may be true for higher negative values of $n$ than -4 , if the sphere have contact of an order higher than the first with the original surface.

It remains to determine the degree of approximation to which the equation (2) is true. The order of the error in this equation is the same as that of the assumption by which this result was obtained, namely that a distribution on the surface of the sphere may be substituted for the shell.

Suppose that, in general, the potential of the shell is represented by the integral

Let

$$
\iiint f(r) d r \sin \theta d \theta d \phi
$$

$$
\int f(r) d r=f_{1}(r)
$$

Then, the limiting values of $r$ being $r=b, r=b+a u$, the potential will be

$$
\begin{aligned}
v & =\int_{0}^{\pi} \int_{0}^{2 \pi}\left\{f_{1}(b+\alpha u)-f_{1}(b)\right\} \sin \theta d \theta d \phi \\
& =\int_{0}^{\pi} \int_{0}^{2 \pi}\left\{\alpha \cdot b f b+\frac{1}{2} \alpha^{2} u^{2} f^{\prime \prime}(b)+\& c .\right\} \sin \theta d \theta d \phi .
\end{aligned}
$$


If this expression be restricted to the first power of $a u$, the expression thus limited, namely

$$
\int_{0}^{\pi} \int_{0}^{2 \pi} \operatorname{auf}(b) \sin \theta d \theta d \phi, \ldots . . .
$$

represents the potential of a superficial distribution whose density is $\alpha u$. This is the form adopted by Laplace. The error in his result is therefore of an order not lower than $\alpha^{2}$, provided that no one of the terms $u^{2} f^{\prime}(b) \sin \theta, u^{3} f^{\prime \prime}(b) \sin \theta, \& c$. become infinite within the limits of integration.

In the present case $f(r)=r^{2}\left(r^{2}+b^{2}-2 b r \cos \theta\right)^{\frac{n+1}{2}}=r^{2}\left\{(r-b \cos \theta)^{2}+b^{2} \sin ^{2} \theta\right\}^{\frac{n+1}{2}}$. Assume $x=r^{2}-b \cos \theta, y=b \sin \theta, z=x^{2}+y^{2}$. Then

$$
f(r)=r^{2} z^{m} \text {, putting } m=\frac{n+1}{2} \text {. }
$$

We have then to inquire whether any term such as

$$
u^{p+1} \frac{d^{p} \cdot r^{2} z^{m}}{d r^{p}} \sin \theta
$$

becomes infinite when $r=b$. But as neither $r^{2}$ or its differential coefficients can become infinite, it will be sufficient to determine the conditions necessary in order that

$$
u^{p+1} \frac{d^{p} z^{m}}{d r^{p}} \sin \theta
$$

may remain finite when $r^{2}=b$, and therefore $\theta=0$. Now, since

we have

$$
\frac{d z}{d r}=2 x \frac{d x}{d r^{r}}=2 x
$$

$$
\frac{d^{p} z^{m}}{d r^{r}}=\mathrm{A} z^{m-q}+\mathrm{B} z^{m-q-1} x^{2}+\mathrm{C} z^{m-q-2} x^{4}+\& c .
$$

or

$$
=\mathrm{A}^{\prime} z^{m-q-1} x+\mathrm{B}^{\prime} z^{m-q-2} x^{3}+\& \mathrm{c} .
$$

according as $p=2 q$ or $=2 q+1$. In both these series, since $z$ and $x$ are both of the order $\theta^{2}$, when $\theta$ is small, it is easily seen that the first term is the term of the lowest order in $\dot{\theta}$. Moreover, as $u=0$ and $\frac{d u}{d \theta}=0$ when $\theta=0$, it is plain that $u$ is also of the order $\theta^{2}$ when $\theta$ is small. Hence when $p=2 q$, the order of the term $u^{p+1-1} \frac{d^{p} z^{m}}{d r^{p}} \sin \theta$ is

$$
4 q+2+2 m-2 q+1=2 q+2 m+3=2 q+n+4 ;
$$


and when $p=2 q+1$, the order is

$$
4 q+4+2(m-q-1)+2+1=2 q+2 m+5=2 q+n+6 .
$$

The term retained in the value of $v,(6)$, corresponds to $p=0$. The order of this term is therefore $=$ order of $\alpha+n+4$, when $\theta$ is small. Hence if $n+4$ be not $<0$, the order of this term must be $=$ or $>$ order of $\alpha$. The order of the next term will $=$ order of $\alpha^{2}+n+6$, and the orders of all succeeding terms will be higher when $\theta$ is small. The degree of the approximation is therefore as has been stated.

The same reasoning which has been applied to the potential will hold also for the resolved attraction. In fact, as we have seen, when $\theta$ is small the orders of the corresponding parts of the potential and of the resolved attraction are the same. Hence :-

If quantities proportional to the square and higher powers of the thickness of the shell be neglected, Laplace's equation is true for all positive values of $\mathrm{n}$, and for all negative values which do not numerically exceed 4.

It will be observed that in the foregoing investigation I have taken Laplace's equation to refer, not to the potentials and attractions of the solids, but to the potential and attraction of the shell included between them. It is upon this consideration that the validity of the proof of Laplace's equation depends for negative values of $n$ numerically greater than 2 and not numerically greater than 4 . For such values of $n$ the differential expressions for the attraction and the potential admit of a true integration for the shell, or its equivalent the material distribution. But these expressions do not necessarily in such cases admit of a true integration for either of the solids. Thus, if $n$ be numerically greater than 2 and not greater than 3 , the differential expression for the attraction does not admit of a true integration; and if $n$ be greater than 3 , neither expression admits of a true integration. In the former case the attraction is infinite, and in the latter both attraction and potential are infinite.

Hence it is evident that the true cause of the failure of Laplace's equation (when it does fail) is that the quantities with which it is concerned cease to be finite. It does not fail for such a shell as Laplace describes, because the thickness of this shell is zero at the attracted point and exceedingly small in the immediate neighbourhood. It results from this construction of the attracting shell, that the potential and resolved attraction remain finite for higher inverse laws of force than they would for an ordinary solid. 